\title{
Facile construction of novel organic-inorganic hybrid microspheres for enzyme immobilization
}

\author{
Jun Ma ${ }^{1, a}$, Shuo Liü, b , Yanhui Chen ${ }^{1, c}$, Jian $\mathrm{Li}^{1, \mathrm{~d}, \text { * }}$ \\ ${ }^{1}$ School of Chemical Engineering and Materials, Tianjin University of Science and Technology, \\ Tianjin 300457, China. \\ amajun_tust@sina.cn, b317526221@qq.com, ${ }^{\mathrm{c}} 147479053 @ q q . c o m,{ }^{d}$ lijian@tust.edu.cn \\ Corresponding Author: Jian Li
}

Keywords: Membrane emulsification, Biomimetic mineralization, Enzyme immobilization.

\begin{abstract}
In this research, a novel and facile method for preparing organic-inorganic hybrid microspheres was developed by a synergy of membrane emulsification and biomimetic mineralization. The gelatin solution (water phase) was extruded through a Shirasu Porous Glass (SPG) membrane, and mixed with the liquid paraffin (oil phase) to form a monodispersed W/O emulsion. After cooling and cross-linking, the organic gelatin microspheres were obtained, and then immersed in to $\mathrm{Na}_{2} \mathrm{SiO}_{3}$ solution, which acted as silicon precursor. Under the catalysis of gelatin, silica coating was formed around the gelatin microspheres, resulting in the final gelatin/silica hybrid microspheres. The as-prepared hybrid microspheres were subsequently utilized for encapsulation of $\beta$-Glucuronidase (GUS). The results indicated that the recycling and storage stability of the immobilized enzyme are all significantly improved.
\end{abstract}

\section{Introduction}

Enzyme immobilization is a promising technology, which not only permits easy recovery and reutilization of the enzyme, but also improves the stability and tolerance to extreme conditions of the enzymes [1,2]. Recently, the entrapment of enzyme in organic-inorganic hybrid microspheres has attracted increasing attention due to its moderate hydrophilicity, controllable transport characteristics and good physicochemical stability [3, 4]. Biomineralization in nature has offered a delicate prototype of inorganic mineral formation in organisms and organic-inorganic composite hydrogels could be fabricated by biomimetic mineralization under rather mild conditions, i.e., low precursor concentration, near-neutral $\mathrm{pH}$ and ambient temperature [5]. Therefore, the biomimetic mineralization have been evolved as tools for the facile synthesis of organic-inorganic hybrid microspheres.

Membrane emulsification, has been known as a promising technology and developed to prepare monodisperse microspheres [6]. The membrane emulsification method shows significantly advantages in contrast with conventional techniques: (1) the size of obtained microspheres is uniform and can be facile controlled; (2) break-up and coalescence between emulsion droplets rarely occurs; (3) the preparation process is mild without high shear force. Generally, membrane emulsification is usually utilized to construct microspheres with organic [7,8] or inorganic [9] materials individually. However, only a few researches have been reported about producing organic-inorganic hybrid microspheres by membrane emulsification.

The important feature of this research is combining membrane emulsification process with biomimetic mineralization method, for the preparation of polymer-inorganic hybrid microspheres. The gelatin microspheres were prepared through a membrane emulsification process, and then induced biomimetic mineralization in $\mathrm{Na}_{2} \mathrm{SiO}_{3}$ solution. The feasibility of the novel gelatin/silica hybrid microspheres for enzyme immobilization was demonstrated using $\beta$-Glucuronidase (GUS) as the model enzyme. 


\section{Materials and methods}

\subsection{Materials.}

Gelatin (Type A from porcine skin), $\beta$-Glucuronidase (GUS, from Saccharomyces cerevisiae), nicotinamide adenine dinucleotide (NADH, grade I, 98\%) were purchased from Sigma-Aldrich. Baicalin (purity $\geq 98 \%$ ) used as substrate was purchased from Sichuan Xieli Pharmaceutical Co. Ltd. All the other chemicals were of analytical reagent grade and purchased from Tianjin Guangfu Chemical Co. Ltd.

\subsection{Preparation of gelatin/silica hybrid microspheres.}

The gelatin microspheres were prepared by a membrane emulsification process. The gelatin powder was dissolved in hot water as the water phase. Oil phase was prepared by dissolving Span 80 in pre-heated liquid paraffin. The water phase was poured into steel tank and pressed through the SPG membrane into the oil phase to form W/O emulsion. After $15 \mathrm{~min}$, the obtained emulsion was rapidly cooled down to $4^{\circ} \mathrm{C}$, following by adding $1 \mathrm{~mL}$ of glutaraldehyde. Solid gelatin microspheres were obtained by gentle stirring for two hours. The gelatin microspheres were collected by filtration and washed with isopropanol and acetone. Then obtained gelatin microspheres were immersed in $\mathrm{Na}_{2} \mathrm{SiO}_{3}$ solution under mild stirring. After $30 \mathrm{~min}$, silica coating microspheres were filtered and washed with water to remove unreacted silicate.

The GUS-containing gelatin microspheres were prepared through a similar process. GUS was dissolved in gelatin solution (final concentration of enzyme was $0.1 \mathrm{mg} / \mathrm{mL}$ ), and then the water phase was obtained. After process of membrane emulsification and silica mineralizing, GUS-containing gelatin/silica hybrid microspheres were obtained.

\subsection{Characterization.}

Scanning electron microscopy (SEM) measurements were conducted using Philips XL30 ESEM and Hitachi S-4800 instrument at an operation voltage of $20.0 \mathrm{keV}$ and $0.7 \mathrm{keV}$. Elemental analysis was performed by energy dispersive spectroscopy (EDS).

Fourier transform infrared (FTIR) spectra of the micropsheres were obtained on a Bruker Tensor-27 spectrometer. Thermo-gravimetric analysis (TGA) of the gelatin/silica hybrid microspheres was performed on a TG $209 \mathrm{~F} 3$ Tarsus thermo gravimetric analyzer by heating to $800^{\circ} \mathrm{C}$ at a rate of $10^{\circ} \mathrm{C} / \mathrm{min}$ under an air atmosphere.

\subsection{Activity assay.}

Bioconversion of baicalin to baicalein is catalyzed by GUS, which had been described in our previous research [3]. The enzyme activities and stabilities were studied and compared by measuring the amount of baicalein produced.

$0.1 \mathrm{~g}$ of GUS-containing gelatin microspheres was used for successive batches of GUS-catalyzed reactions with 10 minutes cycle. After each cycle, the microspheres were recovered by centrifugation and washed by distilled water. The recycling stability of the immobilized GUS was denoted by recycling efficiency as follows (Eq. (1)).

$$
\text { recycling efficiency }(\%)=\frac{\text { enzyme activity in the } n^{\text {th }} \text { cycle }}{\text { enzyme activity in the } 1^{\text {st }} \text { cycle }} \times 100
$$

The free and immobilized enzyme was both stored at $4 \circ \mathrm{C}$ for a certain period of time. The stability was expressed by storage efficiency as follow (Eq. (2)).

$$
\text { storage efficiency }(\%)=\frac{\text { enzyme activity after storage }}{\text { intial enzyme activity }} \times 100
$$




\section{Results and discussion}

\subsection{Fabrication of the gelatin and hybrid microspheres.}

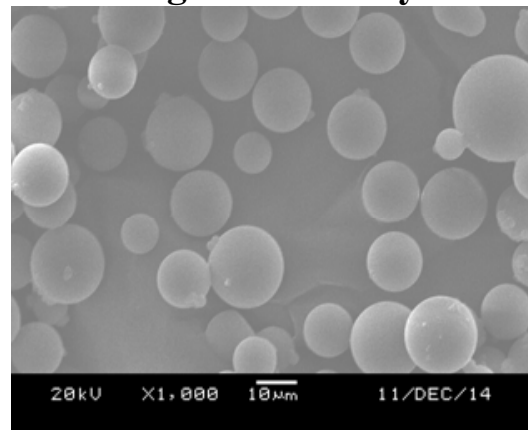

(a)

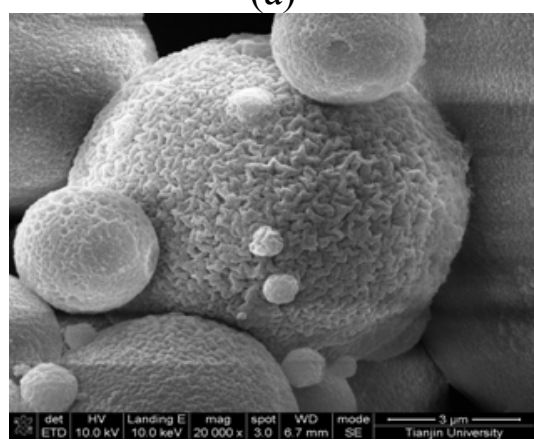

(c)

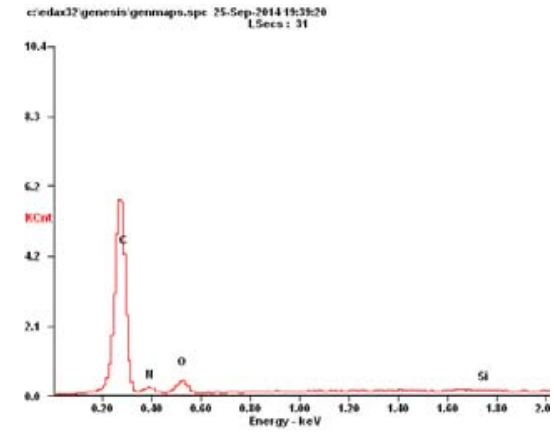

(b)

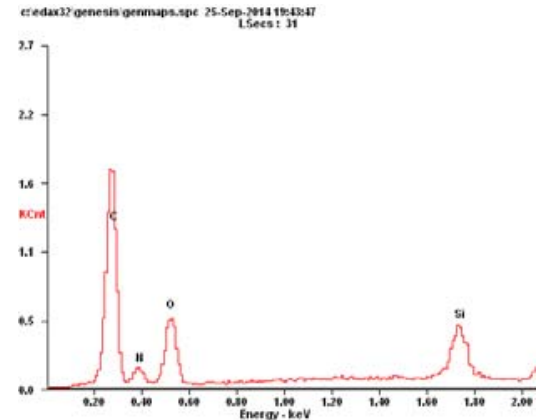

(d)

Fig. 1 SEM images (a) and EDX spectra (b) of gelatin microspheres. SEM images (c) and EDX spectra (d) of gelatin/silica hybrid microspheres.

As shown in Fig.1a, the SEM micrograph presented that the neat gelatin microspheres exhibited not only smooth surface but also a uniform diameter sizes nearly $10 \mu \mathrm{m}$. Fig. $1 \mathrm{~b}$ indicated that $\mathrm{C}, \mathrm{N}$ and $\mathrm{O}$ elements were detective in the surface of gelatin microspheres, which composed of gelatin and glutaraldehyde (cross-linking agent). The morphology of gelatin/silica hybrid microspheres was characterized by SEM (Fig.1c). The hybrid microspheres retained the spherical shape very well and exhibited a rough, integrated and denser surface. The gelatin was able to induce the silica condensation and the subsequent buildup of a cell wall-like inorganic silica layer around the outer surface of the microspheres. The positively charged gelatin could adsorb and concentrated the negatively charged silicon precursor via electrostatic and hydrogen bond interactions. Fig.1d indicated the presence of $\mathrm{C}, \mathrm{N}, \mathrm{O}$ and $\mathrm{Si}$ elements in the hybrid microspheres. Comparing with gelatin microspheres, the weight ratio of $\mathrm{C}$ and $\mathrm{N}$ both decreased but the ratio of $\mathrm{O}$ element increased, while Si element occurred.

\subsection{Characterization of gelatin/silica hybrid microspheres.}

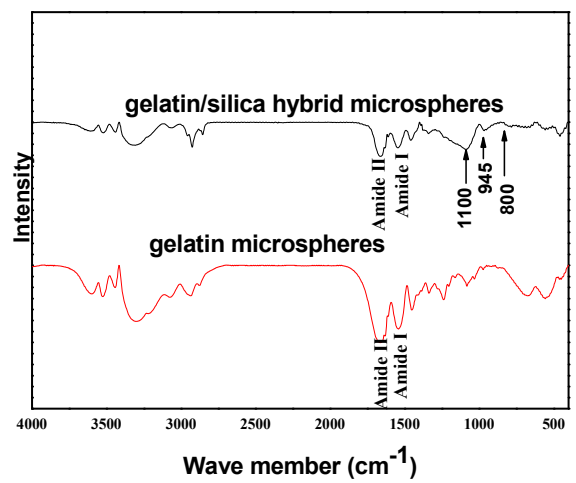

(a)

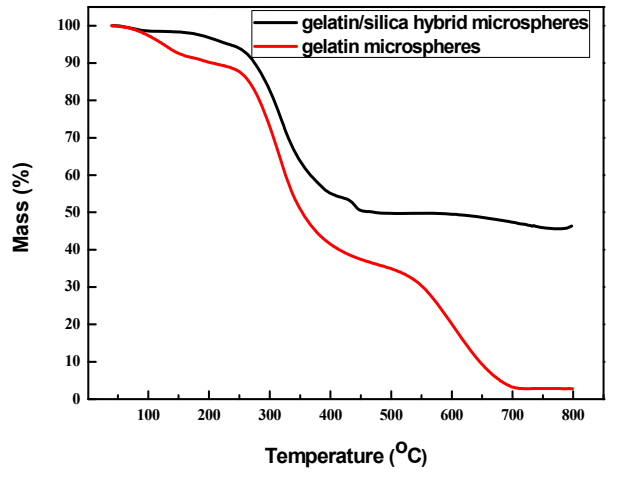

(b)

Fig. 2 FTIR (a) and TGA (b) spectra of microspheres. 
FTIR spectra of GelMs and GelSHMs were compared in Fig. 2a. The band around $3450 \mathrm{~cm}-1$ was correspond to $\mathrm{NH}$ stretching vibrations, and the narrow one near $2920 \mathrm{~cm}-1$ was attributed to $\mathrm{CH}_{2}$ stretching vibrations. The absorption band appeared at $1540 \mathrm{~cm}-1$ and $1650 \mathrm{~cm}-1$ were characteristic bands of Amide I and Amide II [12]. Regarding the gelatin-silica hybrid microspheres, the bands at around $1100 \mathrm{~cm}-1,945 \mathrm{~cm}-1$ and $800 \mathrm{~cm}-1$ were attributed to the Si-O-Si and Si-OH bonds of silica shell [3].

Decomposition profiles of gelatin microspheres and gelatin/silica hybrid microspheres were characterized by Thermogravimetric analysis (TGA), and the results were presented in Fig. 2b. From $50^{\circ} \mathrm{C}$ to $200^{\circ} \mathrm{C}$, above tow samples displayed a weight loss of $10.1 \%$ and $4.6 \%$, respectively. It is related to the loss of adsorbed water. The next step from $200^{\circ} \mathrm{C}$ up to $420^{\circ} \mathrm{C}$ is associated with the helical structure breakage and peptide bonds rupture. At the end of TGA, $46.5 \%$ of hybrid microspheres was not decomposed, which corresponded to the mineralized silica.

\subsection{Catalytic activity and stability of immobilized enzyme.}

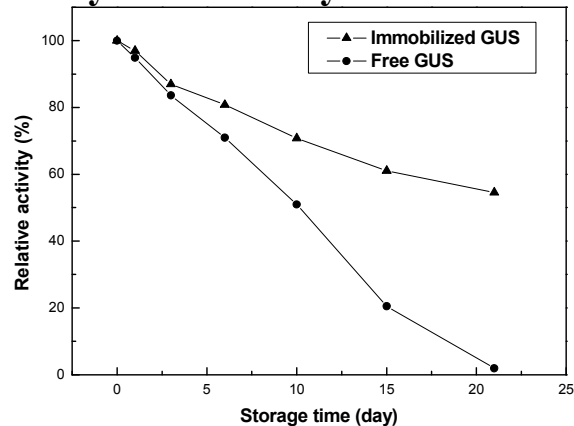

(a)

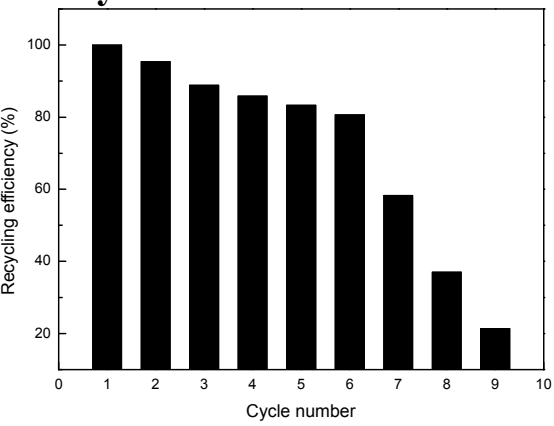

(b)

Fig. 3 The recycling (a) and storage stability (b) of GUS

The storage stabilities of free and immobilized GUS are compared in Fig. 3a. It should be noticed that the storage stability was obviously enhanced after immobilization. During the first 3 days, the activity of immobilized enzyme decreased synchronously with the free one (from $100 \%$ to $\sim 80 \%$ ). From 4 th to 21 st day, the relative activity of free GUS decreased to $2 \%$, while $54 \%$ activity of GUS immobilized in gelatin/silica hybrid microspheres was retained. This result is tentatively explained by unfolding process, which is the main reason of enzyme deactivation during storage [13]. It is supposed that in the initial storage time gelatin/silica hybrid microspheres may allow the unfolding to some extent like it happened in solution, since GUS is immobilized in a hydrophylic, nature-like microenvironment. However, after a certain period of storage, the further unfolding of immobilized GUS will be inhibited by the confinement effect of mesopores. As a consequence, the decrease rate of activity for immobilized GUS become lower than its free form.

The recycling stability of the GUS encapsulated in Gelatin/silica hybrid microspheres was illustrated in Fig. 3b. The GUS encapsulated in Gelatin/silica hybrid microspheres retained more than $80 \%$ of its initial activity after 6th cycles. In 7 th recycling process, the relative activity sharply decreased to $58 \%$, and less than $22 \%$ after 9 cycles. The mineralized silica coating made a significant contribution to increase recycling stability in the initial stage, which might inhibited swelling, suppressed enzyme leakage and improved mechanical strength [14]. However, after multiple recycles under continuous stirring, the gelatin network might become loosen, which could induced enzyme leakage as well as decreasing activity.

\section{Acknowledgement}

The authors thank the financial support from the National Natural Science Foundation of China (21306139), Natural Science Foundation of Tianjin (No. 14JCYBJC20800), and National Undergraduate Training Programs (201510057135). 


\section{References}

[1]. Liya Zhou, Yanjun Jiang, Jing Gao, et al. Oriented immobilization of glucose oxidase on graphene oxide. Biochemical Engineering Journal. Vol.69 (2012), p.28-31.

[2]. Cesar Mateo, Jose M. Palomo, et al. Improvement of enzyme activity, stability and selectivity via immobilization techniques. Enzyme and Microbial Technology. Vol. 40 (2007) No.6, p. 1451-1463.

[3]. Jian $\mathrm{Li}$, Zhongyi Jiang, Hong $\mathrm{Wu}$, et al. Improving the recycling and storage stability of enzyme by encapsulation in mesoporous $\mathrm{CaCO}_{3}$-alginate composite gel. Composites Science and Technology. Vol. 69 (2009) No.3-4, p. 539-544.

[4]. Xinhuang Kang, Jun Wang, Zhiwen Tang, et al. Direct electrochemistry and electro catalysis of horseradish peroxidase immobilized in hybrid organic-inorganic film of chitosan/sol-gel/carbon nanotubes. Talanta. Vol. 78 (2009) No.1, p. 120-125.

[5]. Jian Li, Zhongyi Jiang, Hong Wu, et al. Constructing inorganic shell onto LBL microcapsule through biomimetic mineralization: A novel and facile method for fabrication of microbioreactors. Soft Matter. Vol. 6 (2010) No.3, p.542-550.

[6]. Ma GH. Microencapsulation of protein drugs for drug delivery: Strategy, preparation, and applications. J Control Release. 2014; 193:324-340.

[7]. Wei Y, Wang YX, Wang W, Ho SV, Wei W, Ma GH. mPEG-PLA microspheres with narrow size distribution increase the controlled release effect of recombinant human growth hormone. $\mathrm{J}$ Mater Chem. 2011; 21 (34):12691-12699.

[8]. Zhou QZ, Wang LY, Ma GH, Su ZG. Multi-stage premix membrane emulsification for preparation of agarose microbeads with uniform size. J Membr Sci.2008; 322 (1):98-104.

[9]. Dragosavac MM, Vladisavljevic GT, Holdich RG, Stillwell MT. Production of Porous Silica Microparticles by Membrane Emulsification. Langmuir.

[10]. Staroszczyk H, Pielichowska J, Sztuka K, Stangret J, Kolodziejska I. Molecularand structural characteristics of cod gelatin films modified with EDC and TGase. Food Chem. 2012; 130 (2):335-343.

[11]. Song XK, Wu H, Shi JF, Wang XL, Zhang WY, Ai QH, et al. Facile fabrication of organic-inorganic composite beads by gelatin induced biomimetic mineralization for yeast alcohol dehydrogenase encapsulation. J Mol Catal B-Enzym. 2014; 100:49-58.

[12]. Li J, Wu H, Liang Y, Jiang Z, Jiang Y, Zhang L. Facile Fabrication of Organic-Inorganic Hybrid Beads by Aminated Alginate Enabled Gelation and Biomimetic Mineralization. J BIOMAT SCI-POLYM E. 2013; 24:119-134. 